\title{
Lepromatous Leprosy Mimicking Systemic Lupus Erythematosus
}

\author{
A Clinical Pathology Conference Held by the Division of Rheumatology at Hospital for Special \\ Surgery
}

\author{
Asli Karadeniz, MD • Lindsay Lally, MD • Cynthia Magro, MD • Roger Levy, MD • Doruk Erkan, MD • \\ Michael D. Lockshin, MD
}

Received: 19 January 2014/Accepted: 26 June 2014/Published online: 27 August 2014

(C) Hospital for Special Surgery 2014

Keywords leprosy·anthiphospholipid antibody

\section{Case Presentation}

A 29-year-old Brazilian woman was referred for management of systemic lupus erythematosus (SLE) with antiphospholipid antibodies (aPL). Her symptoms were 1 year of intermittent fever and diffuse, tender, erythematosus, and nodular rash that began during her first pregnancy. She was treated with short course of lowdose corticosteroids, with resolution; however, she suffered an embryonic loss at 7 weeks. Six months prior to admission, she had recurrence of the nodular rash with

Electronic supplementary material The online version of this article (doi:10.1007/s11420-014-9405-9) contains supplementary material, which is available to authorized users.

A. Karadeniz, MD $(\bowtie)$

Department of Internal Medicine, Pendik Training and Research Hospital, Marmara University School of Medicine, Mimar Sinan Caddesi No: 41, Fevzi Cakmak Mah. Pendik, Istanbul, Turkey e-mail: asli_karadeniz@hotmail.com

A. Karadeniz, MD $\cdot$ L. Lally, MD · D. Erkan, MD •

M. D. Lockshin, MD

Department of Rheumatology, Hospital for Special Surgery,

535 East 70th Street, New York, NY 10021, USA

\section{Magro, MD}

Department of Pathology and Laboratory Medicine, Weill-Cornell

New York-Presbyterian Hospital, New York, NY, USA

R. Levy, MD

Department of Rheumatology, Universidade do Estado do Rio de Janerio, Rio de Janerio, Brazil new onset arthralgia; a skin biopsy showed panniculitis. Tests done at that time showed lupus anticoagulant (LA), anticardiolipin antibody (aCL) IgM $>150 \mathrm{U}$ (normal $0-7 \mathrm{U})$, anti- $\beta_{2}$ glycoprotein-I $\left(a \beta_{2} \mathrm{GPI}\right)$ IgM $>150 \mathrm{U}$ (normal $<10 \mathrm{U}$ ), and $\mathrm{a} \beta_{2}$ GPI IgA of $135 \mathrm{U} /$ $\mathrm{mL}$ (normal $<10 \mathrm{U}$ ), the other isotypes being negative. For the presumed diagnosis of a "systemic lupus erythematous (SLE)-like autoimmune disease," she was prescribed hydroxychloroquine (HCQ) $300 \mathrm{mg}$ daily and prednisone $10 \mathrm{mg}$ twice a day with significant response. However, her nodules recurred in 1 month when her prednisone dose was tapered to $15 \mathrm{mg}$ daily, and azathioprine $100 \mathrm{mg}$ daily and dapsone $25 \mathrm{mg}$ daily were begun.

Four months prior to admission, her rash and fever worsened. Prednisone was increased to $60 \mathrm{mg}$ a day with no improvement. Three months prior to admission, she developed symmetric polyarthritis of metacarpophalangeal joints (MCPs), proximal interphalangeal joints (PIPs), wrists, knees, and ankles. Prednisone was increased to $120 \mathrm{mg}$ daily and her azathioprine was switched to mycophenolate mofetil (MMF) $3.0 \mathrm{~g}$ daily with improvement. Two months prior to admission, she was started on enoxaparin sodium subcutaneously $50 \mathrm{mg}$ twice daily. One month prior to admission, MMF was stopped and dapsone dose was increased to $50 \mathrm{mg}$ daily.

Despite the above management, her condition worsened. When she was admitted, she had diffuse erythematous papules and nodules over her extremities, abdomen, chest, back, and face. She denied weight loss, night sweats, alopecia, photosensitivity, oral ulcers, sicca symptoms, Raynaud's phenomenon, chest pain, shortness of breath, cough, nausea and vomiting, abdominal pain, dysuria, hematuria, arthritis, arthralgia, and myalgia. She had no history of thrombosis. She had no recent travel history and had emigrated from 
rural Brazil several years prior to presentation. She denied any alcohol, tobacco, illicit drug use, or new medications. She had Bacillus Calmette-Guérin (BCG) vaccine as a child and had no drug allergies.

On admission, her medications were HCQ $200 \mathrm{mg}$ twice daily, prednisone $20 \mathrm{mg}$ daily, enoxaparin sodium $50 \mathrm{mg}$ twice daily, calcium, vitamin $\mathrm{D}$, and acetylsalicylic acid $325 \mathrm{mg}$ daily. On physical examination, the patient had a temperature of $39.5^{\circ} \mathrm{C}$, heart rate 120 beats per minute, blood pressure $120 / 70 \mathrm{mmHg}$, and oxygen saturation $100 \%$ on room air. She was not ill appearing but had cushingoid features. She had no cervical lymphadenopathy. She had no lower extremity edema, synovitis, or joint tenderness. Skin examination showed livedo reticularis and edematous indurated erythematous papules on her cheeks, forehead, right earlobe, chest, abdomen, and extremities (Figs. 1 and 2). There was no mucosal involvement. The remainder of the examination was normal.

Laboratory evaluation showed marked leukocytosis and mild anemia. Her partial thromboplastin time (PTT) was slightly elevated and she was hypoalbuminemic. She had negative lupus serologies, normal complement levels, and triple aPL-positivity (Table 1). A repeat skin biopsy was performed.

\section{Discussion-Pathology}

The first outside biopsy, which showed inflammatory changes in the subcutaneous fat consistent with panniculitis was not available for our review. There was no special staining performed in this specimen.

The second punch skin biopsy, which was performed at our center, consisted of three fragments (epidermis, dermis, and subcutis), which showed collections of lipidized macrophages with the dermis and subcutaneous fat with a minimal accompanying lymphocytic and neutrophilic infiltrate (Figs. 3 and 4). The zones of lipophage accumulation were modest and exhibited perivascular accentuation. The specimen showed lobular panniculitis, which was composed of aggregates of histiocytes mixed with rare lymphocytes. The cytomorphology of these histiocytes was characteristic for lepromatous leprosy representing the Virchow's histiocyte characterized by mononuclear cells with abundant granular and vacuolated cytoplasm (Fig. 4).

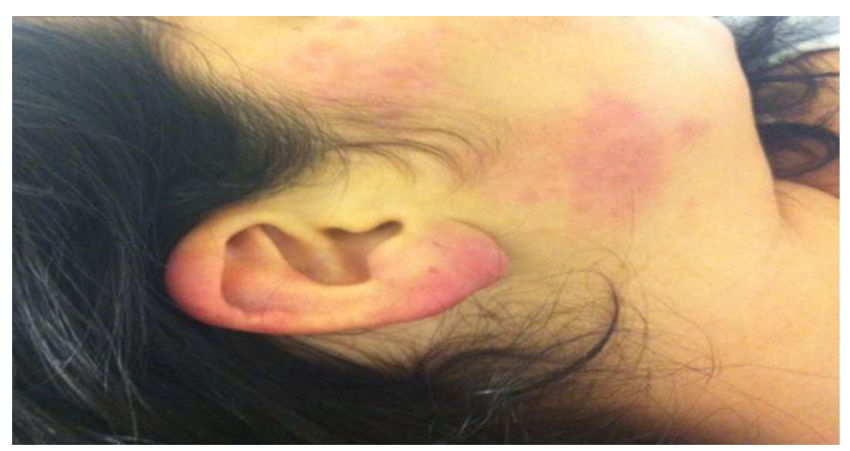

Fig. 1. Diffuse maculopapular erythematous lesions on the face and earlobe.

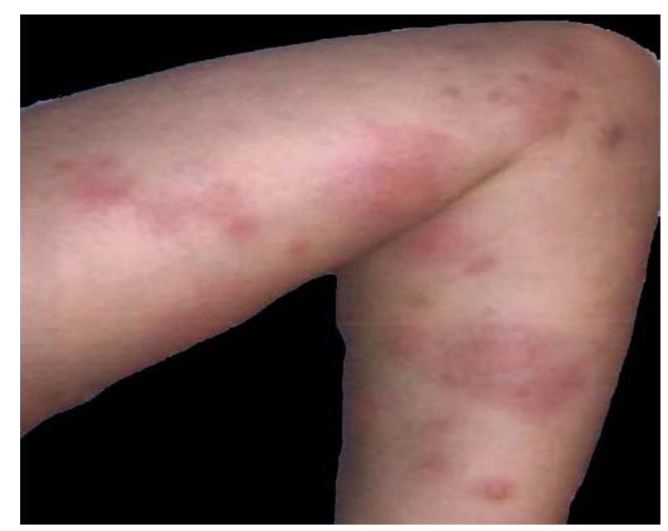

Fig. 2. Diffuse indurated erythematous maculopapular rash on the left arm.

The specimen was stained with a Fite's preparation, a new fuchsin-formaldehyde acid-fast bacilli (AFB) stain for lepra bacilli. At higher magnification, the Fite's stain showed numerous acid-fast positive bacilli within the macrophages (Fig. 5). Well-formed cohesive epithelioid granuloma typical of tuberculoid leprosy was not seen.

Overall these findings were diagnostic of lepromatous leprosy (histiocytoid leprosy) with a minimal adaptive immune response. The clinical presentation along with the morphology captured on this biopsy was typical for lepromatous leprosy.

It is likely that the patient's immunosuppressive therapy resulted in attenuation of the immune response, hence allowing a dysregulated and excessive proliferation of lipid wall containing lepromatous bacilli within scavenger macrophages. These findings were not consistent with any of the lepra reactions. In erythema nodosum leprosum, an Arthus' type 3 immune complex reaction comprising antibody bound to sterile bacterial antigens is manifested morphologically as a leukocytoclastic vasculitis, which was not identified in this case. While there was infiltration of vessel wall, a finding seen in Lucio's phenomenon, there were no supervening inflammatory, septic, or vasculitic changes.

Table 1 Laboratory tests

\begin{tabular}{lll}
\hline Value & Admission & Reference range \\
\hline White blood cell & 27.9 & $3.4-11.2 \mathrm{~K} / \mu \mathrm{L}$ \\
Neutrophils & 93.5 & $45-75 \%$ \\
Hemoglobin & 10.5 & $11.7-16.0 \mathrm{~g} / \mathrm{dL}$ \\
Platelets & 247 & $150-450 \mathrm{~K} / \mu \mathrm{L}$ \\
Prothrombin time & 16.1 & $9.4-11.5 \mathrm{~s}$ \\
Partial thromboplastin time & 60 & $25.0-35.0 \mathrm{~s}$ \\
Antinuclear antibody & Negative & Negative \\
Anti-dsDNA antibody & Negative & Negative \\
Lupus anticoagulant test & 1.89 & $0-1.29 \mathrm{ratio}$ \\
Anti- $\beta_{2}$ glycoprotein I IgM & 150 & $<10.0 \mathrm{U} / \mathrm{mL}$ \\
Anti- $\beta_{2}$ glycoprotein-I IgA & 91 & $<10.0 \mathrm{U} / \mathrm{mL}$ \\
Anti- $\beta_{2}$ glycoprotein I IgG & 5 & $<10.0 \mathrm{U} / \mathrm{mL}$ \\
Anticardiolipin IgG & 2 & $0-14 \mathrm{units}$ \\
Anticardiolipin IgM & 80 & $0-7 \mathrm{units}$ \\
Anticardiolipin IgA & 39 & $0-14 \mathrm{units}$ \\
Complement 3 & 95 & $85.0-193.0 \mathrm{mg} / \mathrm{dL}$ \\
Complement 4 & 20 & $12.0-36.0 \mathrm{mg} / \mathrm{dL}$ \\
\hline
\end{tabular}




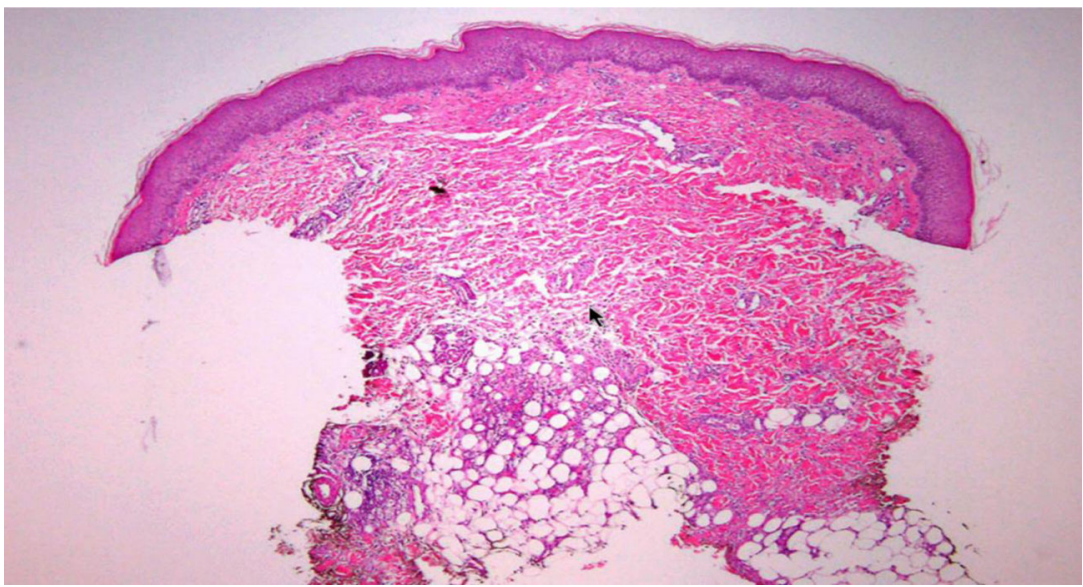

Fig. 3. The skin biopsy shows lipidized macrophages with the dermis and subcutaneous fat with a minimal accompanying lymphocytic and neutrophilic infiltrate.

Of note, panniculitis in the form of erythematous and nodular rash is relatively common in leprosy, occurring in approximately $50 \%$ of patients with lepromatous leprosy, and it reflects a type 2 immunologic reaction (further discussed below) that manifests as eruption of subcutaneous nodules usually in the deep dermis.

\section{Discussion-Rheumatology}

The patient is a 29-year-old Brazilian woman with persistently positive aPL presenting with 1 year of intermittent fever and diffuse tender erythematous nodular rash, which was diagnosed by skin biopsy as lepromatous leprosy.

Leprosy, also known as Hansen's disease, caused by Mycobacterium leprae is an infectious disease preferentially involving the skin and the peripheral nervous system. Brazil is the second in the world in number of cases of leprosy after India. In the Americas, 93\% of the reported cases are from Brazil. Leprosy is more common in men than in women, at a ratio of 1.5 to 1 . The mode of disease transmission is not clear; it is assumed that leprosy mostly spreads by respiratory system or through broken skin; the bacterium has been found in secretions; a long-lasting contact seems to be necessary for transmission [1].

There are four clinical forms of leprosy: indeterminate (early), tuberculoid, dimorphic, and lepromatous (Virchowian). The first two are paucibacillary and the latter two are multibacillary $[17,19]$. The clinical presentation of leprosy is variable including erythema nodosum, arthritis, fever, skin erythema, vasculitis, epididymitis, glomerulonephritis, pericarditis, and pleurisy [17, 19]. Osteoarticular involvement is third most common after the skin and peripheral nervous system manifestations [21]. Lepromatous leprosy causes madarosis (loss of the eyebrows), swelling of the cheeks and earlobes, and disseminated skin nodules on the face that can ulcerate resulting in irreversible damage. The diagnosis is established based on full-depth skin or nerve biopsy smears demonstrating AFB.

Because of diverse clinical manifestations (e.g., dermatological, neurological), leprosy can be confused with other systemic autoimmune diseases including SLE. Thus, physicians should consider leprosy in the differential diagnosis of SLE and/or antiphospholipid syndrome (APS) with unusual

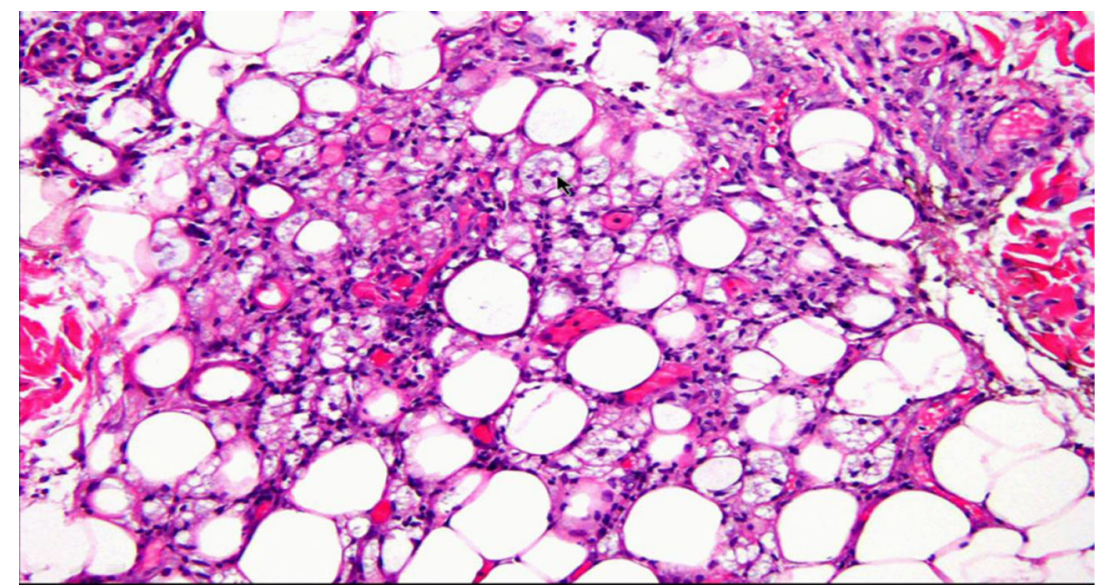

Fig. 4. Panniculitis is composed of aggregates of histiocytes with granular cytoplasm called Virchow cells - the hallmark of lepromatous leprosy mixed with rare lymphocytes. 


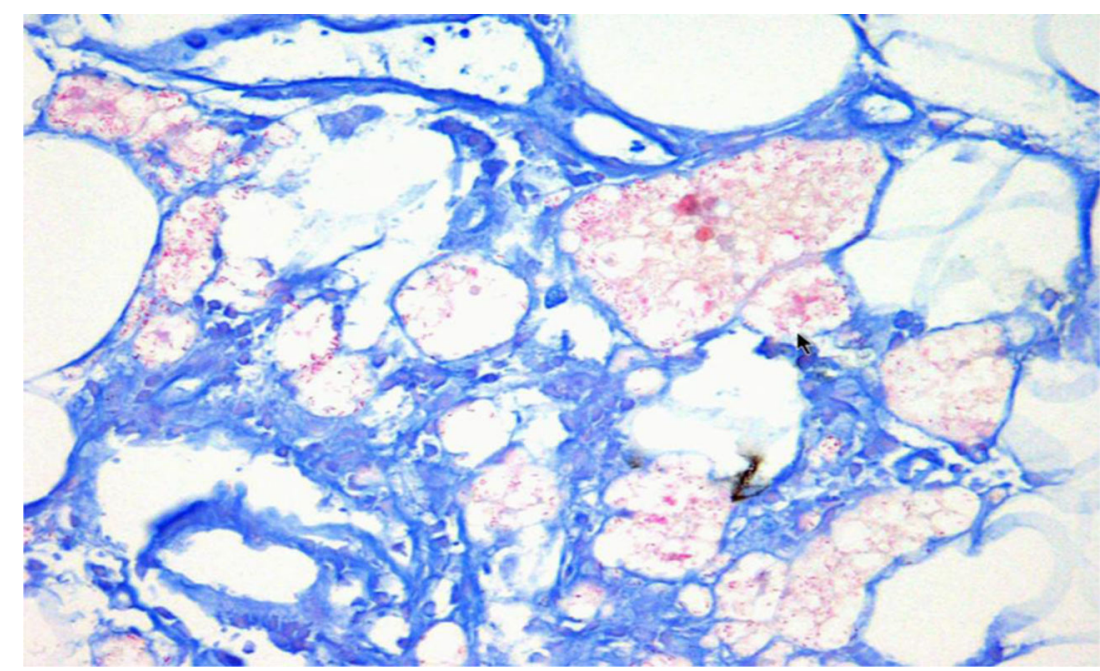

Fig. 5. Fite stain showing bacilli within the vacuolated macrophages.

presentations, especially in endemic areas for leprosy, and order targeted biopsies. Patients can have malar rash, arthritis, photosensitivity, as well as the autoantibodies that can occur in lupus patients [23]. Joint involvement, mimicking rheumatoid arthritis, can also occur in leprosy. A variety of autoantibodies can be detected in leprosy patients such as double-stranded DNA (anti-dsDNA), anti-mitochondrial antibodies (AMA), and aPL [11, 12]. American College of Rheumatology (ACR) lupus classification criteria, which shows sensitivity and specificity of 96 and $84 \%$, respectively, has $16 \%$ false-positive rate for lupus among Brazilian leprosy patients [23].

If therapy for leprosy is delayed, nonreversible damage of the eyes, hands, and feet can occur due to neuropathy [5]. The World Health Organization (WHO) suggests that leprosy should be treated with multiple drug therapy (MDT) to avoid drug resistance. Paucibacillary leprosy should be treated with dapsone and rifampin for 6 months, whereas multibacillary leprosy with dapsone, rifampin, and clofazimine for a year. Other antibiotics like clarithromycin, fluoroquinolones, and minocycline can be used for patients with recurrent disease or for patients who have intolerance to the standard therapy.

Thalidomide, which is used for discoid lupus and myeloma, is also Food and Drug Administration (FDA) approved for erythema nodosum leprosy. This medication is a paradox for rheumatologists because it can induce thrombosis [22], a special concern for aPL-positive patients. It is not clear from the few case reports that if thalidomide induces thrombosis through aPL production or other mechanisms. Some suggest that patients on thalidomide should be anticoagulated or receive lowdose ASA [20].

Bacillus Calmette-Guérin immunization can bolster the immune response in those exposed to leprosy by activating the innate immune response and increasing production of cytokines such as tissue necrosis factor (TNF)-alpha. Thus, there is probably some degree of protection against leprosy in people who have received neonatal BCG vaccination for tuberculosis prevention. As with tuberculosis, the degree of protection conferred by BCG against leprosy should vary from host to host; the reasons for this variability remains unknown.

\section{Discussion-the Association Between Leprosy and Antiphospholipid Antibodies}

In the early $1990 \mathrm{~s}, \beta_{2}$ GPI was discovered as the cofactor for autoimmune aCL as well as the main target antigen for aPL [10]. Early considerations suggested two types of aPL: autoimmune (a $\beta_{2}$ GPI-dependent), and alloimmune or infectious ( $\beta_{2}$ GPI-independent), but this conclusion is currently questioned. $\beta_{2}$ GPI-dependent aCL can rarely occur in some infections, including leprosy $[2,8-10,13,16,18]$.

The prevalence of aPL in leprosy is highly variable; aCL reported in $20-98 \%$ and $a \beta_{2} \mathrm{GPI}$ in $3-89 \%$ of patients $[2,4,8,9,13,14]$. Although IgM is the most common aCL isotype, IgG isotype also occurs, mostly in the lepromatous form [7]. The wide prevalence range of aPL in leprosy patients may be explained by the fact that infection-induced aPL are usually transient, but some leprosy patients develop persistent, possibly autoimmune, aPL.

Lucio's phenomenon is a rare necrotizing skin lesion of leprosy described initially in Mexico. Patients with Lucio's phenomenon develop small vessel thrombosis similar to that seen in APS patients [24]. Biopsies show microthrombosis without inflammation as the abundant bacilli are thought to cause microthrombosis by endothelial proliferation and occlusion. Surprisingly, such patients have a $\beta_{2}$ GPI-dependent autoimmune aPL [15]. Furthermore, Brazilian leprosy patients with Lucio's phenomenon, compared to those without, more commonly fulfill the updated Sapporo Classification Criteria for APS [3].

It is unknown why some leprosy patients develop $\beta_{2}$ GPI-dependent and others $\beta_{2}$ GPI-independent aPL. 
$\beta_{2}$ glycoprotein-I is a single chain glycoprotein consisting of five domains, with domain $\mathrm{V}$ binding to phospholipids. It binds to receptors as a dimmer. Mutation of a domain $\mathrm{V}$ gene that exchanges leucine for valine at position 247 (val247leu in place of val247val) causes structural modification in the protein. A recent study concluded that leprosy patients homozygous for val247val have a greater tendency to produce the pathogenic $\beta_{2}$ GPI-dependent aPL [6].

Leprosy patients may develop a wide spectrum of clinical and laboratory manifestations because of different immunological responses: (a) type 1, seen mostly in tuberculoid leprosy (mild clinical presentation mostly with IgM aCL), represents a slight increase in cell-mediated immunity; and (b) type 2, mostly in lepromatous leprosy (severe clinical presentation mostly with $\operatorname{IgG} \mathrm{aCL}$ ), causes an antigen-antibody complex-mediated immune complex disease with complement activation, the morphologic expression of which is leukocytoclastic vasculitis with expression of TNF- $\alpha$ and interferon- $\gamma$ [14].

\section{Hospital Course}

We speculate that the patient's clinical worsening on lowdose (25-50 $\mathrm{mg}$ daily) dapsone before the leprosy diagnosis was related to her profound concurrent immunosuppressive treatment. Following the diagnosis, the patient's dapsone dose was increased to $100 \mathrm{mg}$ oral daily, and clofazimine $100 \mathrm{mg}$ oral daily and rifampin $600 \mathrm{mg}$ oral daily were added. She showed steady improvement of the next several months, at which time she returned to Brazil and was lost to follow-up.

\section{Conclusion}

Leprosy mimics systemic autoimmune diseases, mainly lupus. In patients from geographic areas in which leprosy is prevalent, leprosy must be included in the differential diagnosis of patients with SLE-like systemic autoimmune diseases and/or aPL with atypical features. Leprosy patients can fulfill the classification criteria for lupus and antiphospholipid syndrome.

\section{Disclosures}

Conflict of Interest: Asli Karadeniz, MD, Lindsay Lally, MD, Cynthia Magro, MD, Doruk Erkan, MD, and Michael D. Lockshin, MD have declared that they have no conflict of interest. Roger Levy, MD reports personal fees and non-financial support from Abbvie and Janssen, personal fees from GSK, Roche, and Pfizer, outside the submitted work.

Human/Animal Rights: All procedures followed were in accordance with the ethical standards of the responsible committee on human experimentation (institutional and national) and with the Helsinki Declaration of 1975, as revised in 2008.

Informed Consent: Informed consent was waived from all patients for being included in the study.
Required Author Forms Disclosure forms provided by the authors are available with the online version of this article.

\section{References}

1. Abraham S, Mozhi NM, Joseph GA, et al. Epidemiological significance of first skin lesion in leprosy. Int J Lepr Other Mycobact Dis. 1998;66: 131-139.

2. Arvieux J, Renaudineau Y, Mane I, et al. Distinguishing features of anti-beta2 glycoprotein I antibodies between patients with leprosy and the antiphospholipid syndrome. Thromb Haemost. 2002;87: 599-605.

3. Azulay-Abulafia L, Pereira Spinelli L, Hardmann D, et al. Lucio phenomenon. Vasculitis or occlusive vasculopathy? Hautarzt. 2006;57: 1101-1105.

4. Baeza I et al. Lepromatous leprosy patients produce antibodies that recognize non-biliary lipid arrangements containing mycolic acids. Mem Inst Oswaldo Cruz. 2012;1: 95-103.

5. Bekri W, Gebre S, Mengiste A, Saunderson PR, Zewge S. Delay in presentation and start of treatment in leprosy patients: A case-control study of disabled and non-disabled patients in three different settings in Ethiopia. Int J Lepr Other Mycobact Dis. 1998;66: 1-9.

6. Brochado MJ, Nascimento MM, Louzada Junior P, Figueiredo JF, Roselino AM. Val247Leu polymorphism of beta2 glycoprotein 1 gene may justify the genesis of anti beta2GP1 antibodies and antiphospholipid syndrome in multibacillary leprosy. An Bras Dermatol. 2009;84: 355-359.

7. de Larrañaga GF, Forastiero RR, Martinuzzo ME, et al. High prevalence of antiphospholipid antibodies in leprosy: Evaluation of antigen reactivity. Lupus. 2000;9: 594-600.

8. Elbeialy A, Strassburger-Lorna K, Atsumi T, et al. Antiphospholipid antibodies in leprotic patients: A correlation with disease manifestations. Clin Exp Rheumatol. 2000;18: 492-494.

9. Forastiero RR, Martinuzzo ME, de Larrañaga GF. Circulating levels of tissue factor and proinflammatory cytokines in patients with primary antiphospholipid syndrome or leprosy related antiphospholipid antibodies. Lupus. 2005;14: 129-136.

10. Galli M, Comfurius P, Maassen C, et al. Anticardiolipin antibodies (ACA) directed not to cardiolipin but to a plasma protein cofactor. Lancet. 1990;335: 1544-1547.

11. Gilburd B, Ziporen L, Zharhary D, et al. Antimitochondrial (pyruvate dehydrogenase) antibodies in leprosy. J Clin Immunol. 1994;14: 14-19.

12. Guedes Barbosa LS, Gilbrut B, Shoenfeld Y, Scheinberg MA. Autoantibodies in leprosy sera. Clin Rheumatol. 1996;15: 26-28.

13. Hojnik M, Gilburd B, Ziporen L, et al. Anticardiolipin antibodies in infections are heterogenous in their dependency on beta 2glycoprotein I: Analysis of anticardiolipin antibodies in leprosy. Lupus. 1994;3: 515-521.

14. Iyer A, Hatta M, Usman R, et al. Serum levels of interferongamma, tumour necrosis factor-alpha, soluble interleukin-6R and soluble cell activation markers for monitoring response to treatment of leprosy reactions. Clin Exp Immunol. 2007;150: 210-216.

15. Levy RA, de Meis E, Pierangeli S. An adapted ELISA method for differentiating pathogenic from nonpathogenic aPL by a beta 2 glycoprotein I dependency anticardiolipin assay. Thromb Res. 2004;114: 573-577.

16. Martinuzzo ME, Forastiero RR, Carreras LO. Anti beta 2 glycoprotein I antibodies: Detection and association with thrombosis. $\mathrm{Br}$ J Haematol. 1995;89: 397-402.

17. Matsuo E. Leprosy as a challenge to science on the ability to decode its enigma. A hypothesis on how to respond. Jpn J Infect Dis. 2005;58: 59-64.

18. McNally T, Purdy G, Machie IJ, et al. The use of an anti-beta 2glycoprotein-I assay for discrimination between anticardiolipin antibodies associated with infection and increased risk of thrombosis. Br J Haematol. 1995;91: 471-473. 
19. Murthy PK. Current epidemiology of leprosy. J Indian Med Assoc. 2004;102: 672-683.

20. Palumbo A, Cavo M, Bringhen S, et al. Aspirin, warfarin, or enoxaparin thromboprophylaxis in patients with multiple myeloma treated with thalidomide: A phase III, open-label, randomized trial. J Clin Oncol. 2011;29: 986-993.

21. Pereira HL, Ribeiro SL, Pennini SN, Sato EI. Leprosy-related joint involvement. Clin Rheumatol. 2009;28: 79-84.
22. Sharma NL, Sharma VC, Mahajan VK, et al. Thalidomide: An experience in therapeutic outcome and adverse reactions. $J$ Dermatol Treat. 2007;18: 335-340.

23. Teixeira Junior GJ, Silva CE, Magalhães V. Application of the diagnostic criteria for systemic lupus erythematosus to patients with multibacillary leprosy. Rev Soc Bras Med Trop. 2011;44: 85-90.

24. Vargas-Ocampo F. Diffuse leprosy of Lucio and Latapí: A histologic study. Lepr Rev. 2007;78: 248-260. 